\title{
Databases and computer resources on plant miRNA to study its role in abiotic stress response
}

\author{
Orlov Y.L. ${ }^{1,2 *}$, Babenko V.N. ${ }^{1,2}$, Dergilev A.V. ${ }^{2}$, Galieva A.G. ${ }^{2}$, \\ Dobrovolskaya O.B. ${ }^{1}$, Chen M. ${ }^{3}$ \\ ${ }^{1}$ Institute of Cytology and Genetics, SB RAS, Novosibirsk, Russia \\ ${ }^{2}$ Novosibirsk State University, Novosibirsk, Russia \\ ${ }^{3}$ Zhejiang University, Hangzhou, China \\ *e-mail:orlov@bionet.nsc.ru
}

Non-coding RNA (ncRNAs) play crucial roles in transcriptional and post-transcriptional regulation of gene expression in plants. New data indicates that ncRNAs, including miRNA and long ncRNA (lncRNAs), have emerged as key regulatory molecules in plant stress responses. Plants are exposed to set of stress factors, such as viral infection, salt, drought, cold, and heat, which limit plant growth and productivity. To adapt and survive plants have utilized multiple gene regulatory mechanisms to restore cellular homeostasis. ncRNAs play a critical role in the regulation of gene expression in response to stress conditions. We review contemporary set of tools and resources to study its role in plant abiotic stress response. Functional RNAs with low protein-coding potential (ncRNAs) are classified as small ncRNA ( $<30 \mathrm{nt})$, medium-sized ncRNA, and long ncRNAs (lncRNAs) (>200 nt). miRNA expression is up-regulated or down-regulated in response to stress similar to changes in expression of protein-coding genes. For example, in Arabidopsis, in response to nitrogen (N) deficiency, expression of miR-160 is upregulated whereas that of miR-169 is downregulated.

Bioinformatics analysis of available sequencing data is important. Several computational tools have been established to identify and predict miRNAs in plants. According to sRNA sequencing (sRNA-seq) data, miRPlant, miRanalyzer, miRA and miRDeep-P could be used to predict new miRNAs. Several databases have been established for archiving miRNAs and their annotation: miRBase collects miRNAs from experimental or computational identification of various species, while Rfam provides miRNA sequences based on homology relationship. Plant miRNA database (PMRD) and Plant microRNA Knowledge Base (PmiRKB) are two well-known plant-specific miRNA annotation databases. Although remarkable progress in studying of miRNAs and lncRNAs role in plant adaption to stress, details are still have to be discovered using new sequencing technologies.

Acknowledgements: This study was supported by the Russian Science Foundation. The authors are grateful to RFBR (18-04-00483) for support of the work. 